\title{
O Sistema de Inovação do Setor Automotivo Brasileiro: lições aprendidas com o INOVAR-AUTO
}

\author{
Ugo Ibusuki (ugoibusuki@usp.br) - Escola Politécnica da USP \\ Paulo Carlos Kaminski (pckamins@usp.br) - Escola Politécnica da USP \\ Erik Telles Pascoal (erik.pascoal@ outlook.com) - Faculdade de Engenharia de Resende
}

\section{RESUMO}

O setor Automotivo Brasileiro é de grande competitividade e responsável por grandes inovações que sustentam o nível tecnológico da engenharia nacional. Por ser composto basicamente de empresas multinacionais, grande parte do desenvolvimento de produtos ocorre em suas matrizes e somente algumas etapas do desenvolvimento ocorrem em suas subsidiárias brasileiras que suportam a criação de conhecimentos locais em Pesquisa e Desenvolvimento (P\&D). Através deste estudo, objetiva-se realizar uma análise crítica do Sistema de Inovação do setor automotivo sob o regime do Programa de Incentivo à Inovação Tecnológica e Adensamento da Cadeia Produtiva de Veículos Automotores (INOVAR-AUTO), vigente de 2013 a 2017, que definiu metas mandatórias de eficiência energética e dispêndios em P\&D e Engenharia. A metodologia de pesquisa utilizada foi a qualitativa e exploratória através de entrevistas com os principais players do sistema de inovação do setor automotivo (empresas, universidades, ICTs). Os resultados demonstram que as montadoras já estabelecidas há mais tempo no país possuem uma engenharia local robusta e níveis de investimentos em $\mathrm{P} \& \mathrm{D}$ e Engenharia que superam os valores definidos pelo INOVAR-AUTO. Em contrapartida, os novos entrantes foram obrigados a investirem em um valor mínimo de engenharia local definido pelo programa. No entanto, devido a falta de metas claras para um plano tecnológico do setor automotivo brasileiro, boa parte do valor investido pelas montadoras entrantes foi canalizada para a criação de laboratórios e centros de pesquisa sem uma definição visível do propósito de longo prazo em termos de qual seria o diferencial competitivo do setor automotivo brasileiro no que tange a $P \& D$ e Engenharia.

Palavras-chave: Inovação, Sistema Nacional de Inovação, Políticas Públicas, Setor Automotivo Brasileiro, Inovar-Auto

Área: Inovação e a gestão do desenvolvimento de produtos e serviços em empresas no Brasil

\section{INTRODUÇÃO}

A indústria automotiva brasileira, apesar da crise recente, confirma sua posição de ser um dos setores mais importantes da economia nacional e internacional. Segundo a ANFAVEA Associação Nacional dos Fabricantes de Veículos Automotores (2016), no ano de 2016 foram 
produzidos 2.1 milhões de veículos automotores. O setor representa hoje quase $22 \%$ do PIB industrial e 5\% do PIB total, com faturamento acima de R \$150 bilhões no ano de 2016.

O parque industrial automotivo brasileiro é composto de 31 fabricantes, 500 autopeças e 5.386 concessionárias. No total reúnem 64 unidades industriais distribuídas em 10 estados brasileiros. Em quantidade de empregos diretos, a indústria automotiva emprega 130 mil pessoas. $\mathrm{O}$ montante total de geração de empregos diretos e indiretos de toda a cadeia automotiva representa 1.5 milhões de pessoas (ANFAVEA, 2016).

No âmbito internacional, a indústria automotiva brasileira é atualmente o $10^{\circ}$ produtor mundial e $08^{\circ}$ mercado consumidor (OICA - International Organization of Motor Vehicle Manufacturers, 2016). Em relação a volumes de investimentos, até o ano de 2017 estão previstos investimentos da ordem de $\mathrm{R} \$ 85$ bilhões destinados à ampliação, modernização e construção de novas fábricas e desenvolvimento de novos produtos incentivados principalmente pelo novo regime automotivo INOVAR-AUTO (MDIC, 2012). Apesar destes investimentos, as perspectivas de mercado não são tão animadoras revelando uma queda de $25 \%$ na demanda de automóveis para o acumulado de 2015 e gerando uma situação de capacidade ociosa e excesso de mão-de-obra contratada.

Apesar do progresso nos últimos anos, a balança comercial da indústria automotiva brasileira registra, desde 2009, um forte crescimento nas importações de veículos e autopeças, conforme apresentado nas figuras 1 e 2, com alguma melhora após a introdução do INOVAR-AUTO.

Estes números demonstram claramente um avanço forte dos concorrentes estrangeiros no mercado automotivo brasileiro e, de acordo com IBUSUKI et.al. (2015) coloca em alerta o futuro e a competitividade de toda a cadeia automotiva e afeta sua capacidade local de projetar as autopeças, os sistemas e os produtos finais. Outro ponto importante é que apesar do setor automotivo brasileiro ser responsável por grandes investimentos que sustentam o nível tecnológico da engenharia nacional, o desenvolvimento de produtos locais é, em sua maioria, apenas adaptações de produtos desenvolvidos pelas matrizes localizadas em países desenvolvidos, consequentemente o nível de inovação é baixo, com pouca pesquisa local.

Dentro deste contexto, o governo brasileiro aprovou em Outubro de 2012 o novo regime automotivo INOVAR-AUTO (MDIC, 2012). Este programa tem um foco de longo-prazo (2013 a 2017) e engloba os seguintes objetivos: proteção da indústria local e do mercado doméstico, aumento do investimento em inovação e melhoria da eficiência energética da frota nacional. Sem dúvida, a indústria automotiva brasileira vive tempos de novos desafios e mudanças e, inicia-se um novo ciclo com o regime automotivo para o período 2013-2017.

Através deste estudo, objetiva-se realizar uma análise crítica do sistema de inovação do setor automotivo sob a ótica do regime automotivo INOVAR-AUTO, analisando seus efeitos na política de investimento em P\&D e Engenharia das montadoras estabelecidas no Brasil identificando se houve algum estímulo para o incremento de P\&D e Engenharia local. O principal propósito deste trabalho consiste em coletar lições aprendidas a fim de contribuir para a elaboração de futuras políticas públicas para o setor automotivo brasileiro, no momento em discussão junto as entidades da categoria e o MDIC, denominado de ROTA2030, com prazo para entrar em vigor apartir de 2018. 


\section{Congresso Brasileiro de Inovação e Gestão de \\ Desenvolvimento do Produto}

04 e 05 de setembro de 2017 - Escola Politécnica da Universidade de São Paulo

Figura 1: Balança Comercial da Indústria Automotiva - 2009/2015

Fonte: Adaptado ANFAVEA (2016)

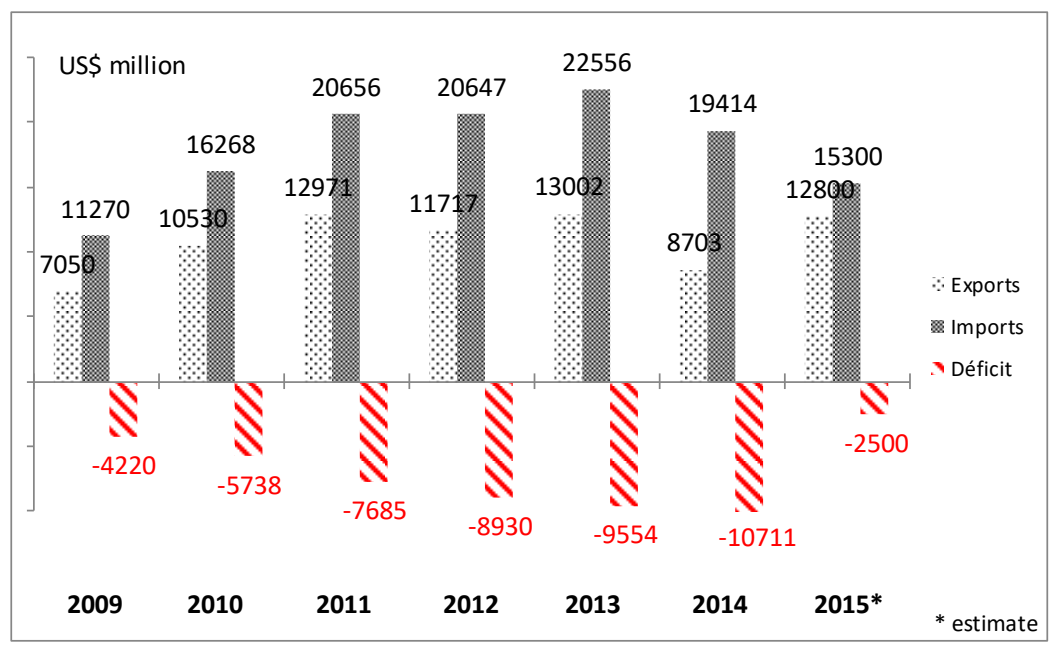

Figura 2: Balança Comercial da Indústria de Auto-peças - 2009/2015

Fonte: Adaptado SINDIPEÇAS (2016)

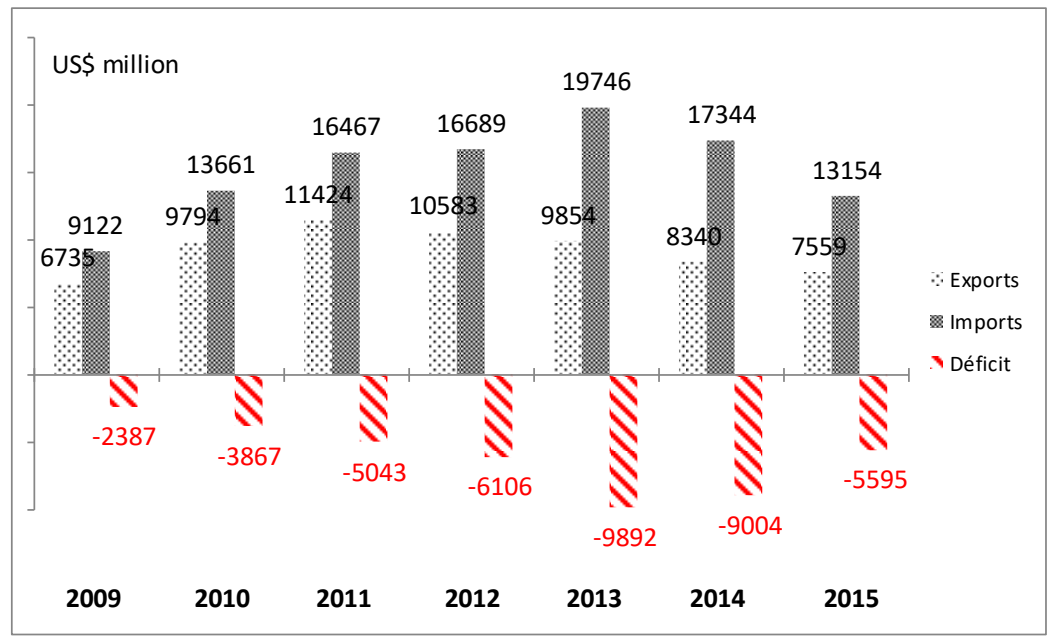

A estrutura deste artigo está dividida da seguinte forma: na seção 1 é apresentada uma introdução geral caracterizando o setor automotivo brasileiro e os objetivos do artigo; na seção 2 é mostrada uma análise do sistema nacional de inovação destacando o sistema de inovação do setor automotivo brasileiro e o Programa INOVAR-AUTO; na seção 3 é apresentado o objetivo e o resultado da pesquisa de campo; e por fim a seção 4 é dedicada às considerações finais e conclusões do artigo.

\section{O SISTEMA NACIONAL DE INOVAÇÃO}

Um Sistema Nacional de Inovação é um grupo articulado de instituições dos setores público e privado (agências de fomento e financiamento, instituições financeiras, empresas públicas e 
04 e 05 de setembro de 2017 - Escola Politécnica da Universidade de São Paulo

privadas, instituições de ensino e pesquisa, etc.) cujas atividades e interações geram, adotam, importam, modificam e difundem novas tecnologias, sendo a inovação e o aprendizado seus aspectos cruciais. É o nível de articulação entre os diversos atores que compõem um SNI que determina a capacidade em gerar inovação. $O$ sistema nacional de inovação e suas instituições desempenham um papel crítico de sustentação às atividades do setor industrial, ao tentar assegurar a coesão das ações governamentais com os objetivos de garantir as estratégias de competitividade e inovação das empresas caracterizadas por projetos tecnológicos de ciclos de produto e processos longos, critérios mais elevados de complexidade, valor adicionado e incerteza (LEMOS et.al., 2010). Nesse sentido, o grau de maturidade e dinamismo dos sistemas nacionais de inovação tendem a assegurar a concretização destes projetos pela oferta de serviços de $\mathrm{P} \& \mathrm{D}$, redução dos riscos, condições diferenciadas para o desenvolvimento tecnológico e comercialização de produtos, a partir dos quais as empresas podem se estruturar para obterem condições de competitividade mais equânimes e sustentáveis no mercado mundial (ABDI, 2010).

\subsection{O Sistema de Inovação do Setor Automotivo Brasileiro}

O Sistema de Inovação do setor automotivo brasileiro, ilustrado na figura 3, demonstra um baixo nível de cooperação e interação entre os vários agentes dos sistemas da inovação incluindo: Institutos de Ensino, Instituto de Pesquisa, Instituto Financeiro, Governo e Associações.

O processo de inovação está fortemente relacionado à hierarquia matriz-subsidiária da montadora (OEM - Original Equipment Manufacturer) e também da relação matriz-subsidiária das autopeças através de transferência de tecnologia existente normalmente em sua matriz. Os fornecedores de nível 1 (Tier 1 ) desenvolvem e industrializam as adaptações de tecnologia para aplicação local atuando diretamente com as montadoras locais. Este processo é o principal gerador de conhecimento e inovações, porém, em sua grande maioria adaptativa. Geralmente os fornecedores de nível 1 são empresas globais que já possuem uma relação de parceria industrial com a montadora em sua matriz e fazem a localização com as subsidiárias brasileiras através do "follow-sourcing" que é a política de trabalhar com o mesmo fornecedor de um item em todas as suas unidades produtivas, independente da localização geográfica dessas unidades.

No entanto para desenvolver a localização em diferentes regiões, o fornecedor de nível 1 precisa atuar com os fornecedores níveis 2 e 3 (Tier 2 e 3 ) da cadeia de autopeças que são normalmente empresas de capital nacional. Este processo de desenvolvimento e industrialização gera conhecimento local para adaptação de componentes e matéria-prima de acordo com as especificidades locais de aplicação e disponibilidade (IBUSUKI et.al., 2012). 
Figura 3: Sistema de Inovação do Setor Automotivo Brasileiro

Fonte: adaptado de IBUSUKI et.al. (2012)

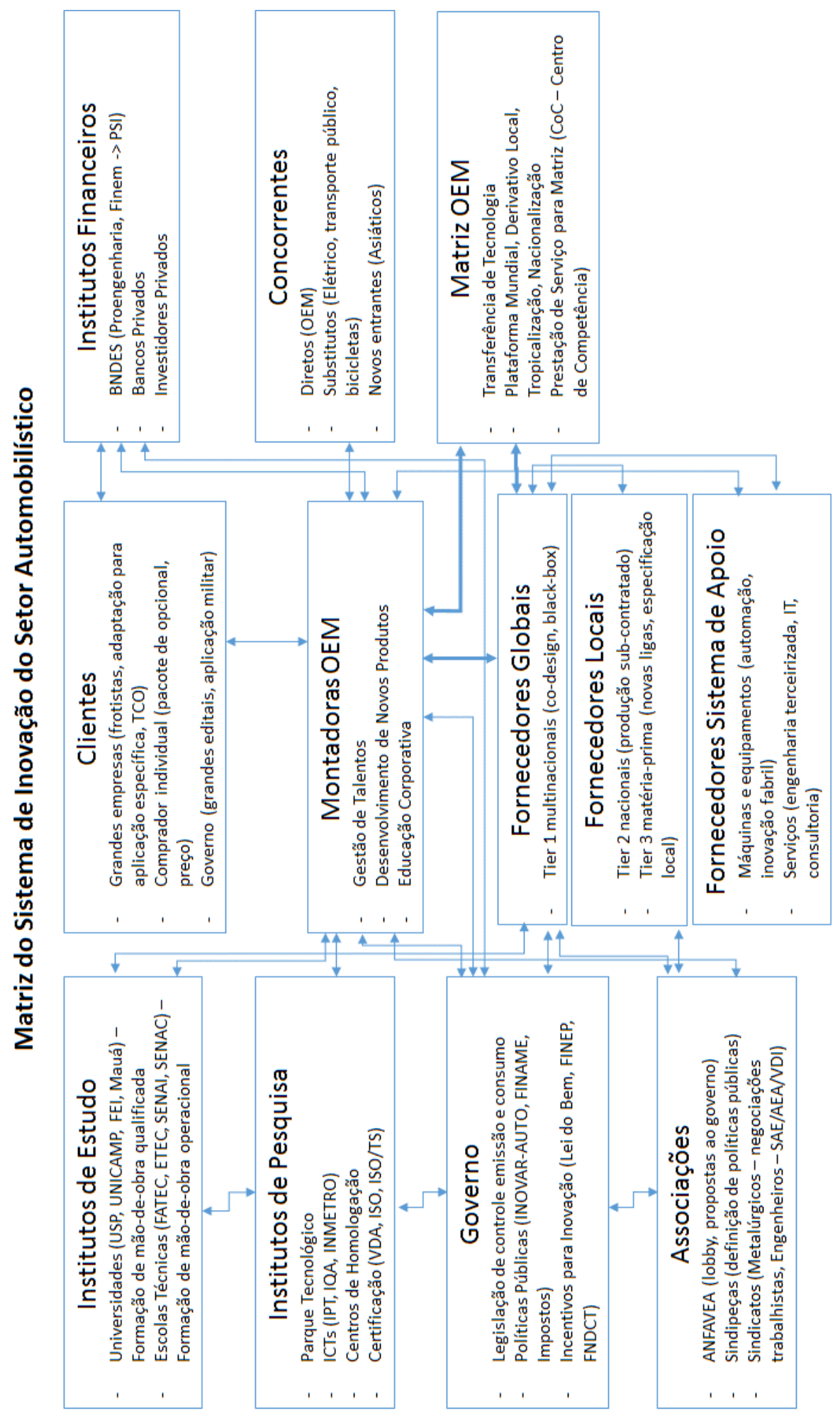




\subsection{O INOVAR-AUTO}

De acordo com a Lei No 12.715 (BRASIL, 2012), o INOVAR-AUTO tem como objetivo expresso de elevar o patamar tecnológico associado aos produtos e aos processos da indústria automotiva nacional por meio das seguintes medidas:

- Criação de condições de competitividade;

- Fortalecimento da cadeia nacional de fornecedores e aumento do conteúdo regional dos veículos produzidos no Brasil medido pelo volume de aquisições de peças e insumos;

- Aumento da eficiência energética dos veículos (carros mais econômicos) e inserção da indústria automotiva do País na rota tecnológica global;

- Investimentos em pesquisa, desenvolvimento e inovação (P,D\&I);

- Aumento do volume de gastos em engenharia, tecnologia industrial básica (TIB) e capacitação de fornecedores.

O Inovar-Auto concede benefício fiscal às empresas habilitadas sob a forma de crédito presumido de IPI que será apurado com base nos investimentos/gastos realizados em cada mêscalendário relativos a: Insumos estratégicos; Ferramentaria; Pesquisa; Desenvolvimento tecnológico; Inovação tecnológica; Recolhimentos ao Fundo Nacional de Desenvolvimento Científico e Tecnológico (FNDCT); Capacitação de fornecedores e; Engenharia e tecnologia industrial básica.

Além disto, as empresas deveriam cumprir um conjunto de requisitos gerais e específicos conforme detalhados na figura 4.

Figura 4: Percentuais mínimos dos requisitos de habilitação ao INOVAR-AUTO

Fonte: PASCOAL (2015)

\begin{tabular}{|c|c|c|c|c|c|c|}
\hline \multirow{2}{*}{ Ano } & \multicolumn{3}{|c|}{$\begin{array}{l}\text { Quantidades mínimas de atividades } \\
\text { fabris e de infraestrutura de } \\
\text { engenharia }\end{array}$} & \multirow{2}{*}{$\begin{array}{c}\text { Percentuais mínimos } \\
\text { de investimentos em } \\
\text { pesquisa e } \\
\text { desenvolvimento }\end{array}$} & \multirow{2}{*}{$\begin{array}{c}\text { Percentuais } \\
\text { mínimos de } \\
\text { investimentos em } \\
\text { engenharia, } \\
\text { tecnologia } \\
\text { industrial básica } \\
\text { e capacitação de } \\
\text { fornecedores* }\end{array}$} & \multirow{2}{*}{$\begin{array}{c}\text { Percentuais } \\
\text { mínimos de } \\
\text { produtos para } \\
\text { adesão ao } \\
\text { programa de } \\
\text { Etiquetagem } \\
\text { Veicular** }\end{array}$} \\
\hline & $\begin{array}{l}\text { Automóveis e } \\
\text { comerciais } \\
\text { leves }\end{array}$ & Caminhões & $\begin{array}{l}\text { Chassis } \\
\text { com } \\
\text { motor }\end{array}$ & & & \\
\hline 2013 & 8 & 9 & 7 & $0,15 \%$ & $0,50 \%$ & $36 \%$ \\
\hline 2014 & 9 & 10 & 8 & $0,30 \%$ & $0,75 \%$ & $49 \%$ \\
\hline 2015 & 9 & 10 & 8 & $0,50 \%$ & $1,00 \%$ & $64 \%$ \\
\hline 2016 & 10 & 11 & 9 & $0,50 \%$ & $1,00 \%$ & $81 \%$ \\
\hline 2017 & 10 & 11 & 9 & $0,50 \%$ & $1,00 \%$ & $100 \%$ \\
\hline
\end{tabular}




\section{OBJETIVO DA PESQUISA DE CAMPO}

A pesquisa de campo teve como objetivo analisar os efeitos do INOVAR-AUTO na política de investimento em P\&D e Engenharia das montadoras estabelecidas no Brasil identificando se houve algum estímulo para o incremento de P\&D e Engenharia local.

Foram realizadas entrevistas com pelo menos um executivo de nível gerencial de cada montadora do setor automotivo brasileiro, professores e pesquisadores relacionados ao setor automotivo, tais como Poli-USP, FEI, Faculdade de Engenharia Mauá, SENAI, Embrapii, FINEP, ABDI. As entrevistas foram estruturadas de forma aberta, direcionando apenas a questão dos efeitos do INOVAR-AUTO para o setor automotivo brasileiro.

Agrupamos as empresas em três grupos distintos conforme o período em que a empresa foi estabelecida no mercado brasileiro:

a) First comers: VW, GM, Fiat, Ford (montadoras estabelecidas no Brasil antes da abertura de mercado em 1990 com o Mercosul);

b) Late comers: Toyota, Honda, Renault, PSA, Hyundai, Nissan (montadoras que se instalaram no Brasil após a abertura de mercado);

c) New comers: Chery, JAC, BMW, Jaguar, Mercedes-Benz (montadoras que se instalaram recentemente com o novo regime automotivo INOVAR-AUTO).

\section{RESULTADO DA PESQUISA DE CAMPO}

Com base nas entrevistas e levantamento de dados em jornais e revistas especializadas do setor automotivo brasileiro, foi possível agrupar a estratégia de investimento em P\&D e Engenharia destas montadoras da seguinte forma:

a) First comers: já possuem uma engenharia de aplicação robusta para nacionalização e tropicalização de componentes, além de desenvolvimento de plataformas e derivativos locais. Os dispêndios em P\&D e Engenharia destas empresas estão próximos do Triad Market (2 3\%). No entanto, devido às exigências de investimentos mínimos em $\mathrm{P} \& \mathrm{D}$ e Engenharia definidas pelo INOVAR-AUTO $(0,5 \%$ e $1 \%$ respectivamente) estarem em patamares inferiores aos investimentos já realizados por estas montadoras, muitas delas adotaram plataformas globais que atendem às exigências de eficiência energética do INOVAR-AUTO com consequente decomposição da engenharia local. Ou seja, o INOVAR-AUTO trouxe indiretamente redução de engenharia local. Como exemplo, podemos citar a aplicação do motor 1.0 turbo do Volkswagen Up TSI, que estreou aqui antes de ser lançado na Europa; o motor 3 cilindros da Ford já disponível na matriz adaptado para motores flex (bicombustível); a GM que deixou de ser um centro de competência mundial em picapes médio devido a queda de volume local neste segmento; a exceção foi a Fiat que estabeleceu projetos de pesquisa junto a universidades para redução de consumo, peso e atrito com o objetivo de atingir as metas de eficiência energética. 
b) Late comers: para atender aos requisitos do INOVAR-AUTO, estas empresas focadas somente na produção local de plataformas globais, tiveram que alterar sua estratégia local investindo em P\&D e Engenharia local. Inauguraram centros de pesquisa com foco em nacionalização e tropicalização, além de laboratórios para controle de emissão dos motores. Outros desenvolveram centros de design para adaptação das plataformas globais ao "gosto brasileiro" focando no segmento de alto crescimento local (SUVs e veículos off-road). Como exemplo, podemos citar a PSA com investimento de aproximadamente $\mathrm{R} \$ 30$ milhões para a construção de laboratório de emissões veiculares na Fábrica de Porto Real (RJ), destinado a testar dentro da fábrica o nível de emissão de poluentes de seus veículos; Hyundai realizou investimento estimado de R \$ 100 milhões para construção de centro de pesquisa e desenvolvimento na fábrica de Piracicaba (SP) com o objetivo inicial de desenvolver motores flex; as montadoras japonesas que estabeleceram centros de desenvolvido local para tropicalização e desenvolvimento de derivativos locais (e.g. Honda WR-V; Toyota Etios Cross, Nissan Kicks Rio version).

c) New comers: basicamente composto por empresas de veículos premium e de origem chinesa, tiveram que estabelecer novas plantas para se beneficiarem do crédito presumido do IPI. São empresas ainda com baixa escala de produção quase em regime de produção CKD (complete knocked-down). Tiveram pouco tempo para estabelecer uma estratégia de investimento em $\mathrm{P} \& \mathrm{D}$ e Engenharia local portanto basicamente realizaram investimento em laboratórios de fábrica (para controle dimensional e de material) e aportaram verba no FNDCT proporcional ao déficit para atingimento da meta estipulada pelo INOVAR-AUTO.

\section{CONCLUSÃO}

Com base nos dados da pesquisa, podemos concluir que apesar do INOVAR-AUTO ser uma política de incentivo a $\mathrm{P} \& \mathrm{D}$ e Engenharia local, até o momento não foi possível verificar um aumento expressivo nos dispêndios das empresas do setor automotivo neste campo, além de não estimular maior interação entre os atores do sistema da inovação. Uma das hipóteses que justifica este fenômeno é de que o programa não possuir regras claras e objetivas para direcionar o desenvolvimento tecnológico no setor automotivo nacional. Apesar de possuir metas claras de eficiência energética, o programa não diferencia os veículos mais eficientes de tecnologia avançada como elétrico ou híbrido e nem mesmo os veículos movido a combustível alternativo como etanol e biodiesel com base tecnológica nacional (MARX e MELLO, 2014). Portanto as empresas em sua maioria multinacionais do setor automotivo, meramente aplicam suas cotas de investimento mandatório em adaptação de sistemas de eficiência energética já disponíveis em suas matrizes, o que não gera um desenvolvimento em P\&D e Engenharia local, ao contrário, incentiva somente a industrialização de tecnologia já disponível em suas matrizes. Apesar do INOVAR-AUTO prever a distinção de P\&D e Engenharia, com metas distintas para ambos, quase todas as montadoras optaram pelo atingimento da meta somente de Engenharia (2 metas mandatórias de 3 optativas: $P \& D$, Engenharia e Etiquetagem veicular), que engloba 
investimentos em fábrica e manufatura, facilitando o seu atingimento sem a necessidade de maiores investimentos em P\&D.

Como sugestão de melhoria de uma política de inovação do setor automotivo brasileiro pós INOVAR-AUTO, deve se definir regras claras de longo prazo com definição do objetivo tecnológico setorial para diferencial competitivo, por exemplo motores movido a combustível alternativo (etanol, biodiesel etc). Com isso poderia direcionar investimento mandatório em um objetivo comum entre as montadoreas, por exemplo um centro de pesquisa automotiva que fomentaria o P\&D junto a cadeia de auto-peças (PMEs), universidades e ICTs, incorporando o setor automotivo brasileiro na cadeia global de valor como provedor de motores de combustível alternativo.

\section{PROPOSTAS PARA ESTUDOS FUTUROS}

Este artigo consiste num resultado preliminar de um estudo de pós-doutorado com suas limitações de um trabalho parcial. Além disso, devido ao INOVAR-AUTO ainda estar em vigência até o final do ano, quando as montadoras submeterão por um processo de auditoria pelo MDIC para comprovar o atingimento das metas mandatórias, muitas informações não estão disponvéis para o público em geral, até mesmo por questões de confidencialidade.

Com isso, as limitações consistem na falta de uma análise quantitativa para embasar a conclusão do estudo, sendo esta baseado somente em análise qualitativa, resultado das entrevistas com os agentes do sistema da inovação do setor automotivo brasileiro. Sugestões para estudos futuros consistem no levantamento de dados que corroboram efetivamente com a conclusão de que não houve avanços significativos em $\mathrm{P} \& \mathrm{D}$, cruzando números antes e depois do INOVAR-AUTO que poderiam mostrar, que os resultados não são satisfatórios.

\section{REFERÊNCIAS}

ABDI - Relatório Setorial: Determinantes da Acumulação de Conhecimento para Inovação tecnológica nos Setores Industriais no Brasil - Base Industrial de Defesa, Brasília, 2010.

ANFAVEA - Brazilian Automotive Industry Association, Brazilian Automotive Industry Yearbook, São Paulo (2016). Available in http:// www.anfavea.com.br.

BRASIL. Lei n. 12.715, de 17 de setembro de 2012. Institui o Programa de Incentivo à Inovação Tecnológica e Adensamento da Cadeia Produtiva de Veículos Automotores, e dá outras providências. Diário Oficial da União, Brasília, seção 1, p. 1, 2012. Disponível em: <http://www.lexml.gov.br/urn/urn:lex:br:federal:lei:2012-09-17;12715>.

IBUSUKI U., BERNARDES R. C., CONSONI F. New Brazilian automotive industrial policy: analysis of the consequences for local R\&D based on new comer's strategies. International Journal of Automotive Technology and Management, Vol. 15, No. 1, pp 63-79, 2015.

IBUSUKI U., KOBAYASHI H., KAMINSKI P.C. Localization of product development based on competitive advantage of location and government policies: a case study of car makers in 
Brazil, International Journal of Automotive Technology and Management, Vol. 12, No. 2, pp. 172-196, 2012.

LEMOS M.B., DE NEGRI J.A., RIBEIRO L.C., RUIZ R., ALBURQUERQUE E. Fundos setoriais e sistema nacional de inovação: uma avaliação exploratória, Relatório 1, Projeto: Metodologia de avaliação dos resultados de conjuntos de projetos apoiados por Fundos de Ciência e Tecnologia e Inovação (C, T\&I), 2010.

MARX R., MELLO, A. M. New initiatives, trends and dilemmas for the Brazilian automotive industry: the case of Inovar Auto and its impacts on electromobility in Brazil. International Journal of Automotive Technology and Management, Vol. 14, No. 2, pp. 138-157, 2014.

MDIC - Brazilian Ministry of Development Industry and Commerce, law no 12.715. (2012a). Available in http://www.planalto.gov.br/ccivil_03/_ato2011-2014/2012/lei/112715.htm.

OICA - International Organization of Motor Vehicle Manufacturers, World motor vehicle production by country and type (2016). Available in http://www.oica.net.

PASCOAL E.T., Novo Regime Automotivo Brasileiro: desafios e oportunidades da região sul fluminense, Tese de doutorado apresentada à Faculdade de Engenharia do Campus de Guaratinguetá, Universidade Estadual Paulista (UNESP) Guaratinguetá, 2015.

SINDIPEÇAS - Brazilian Auto Parts Industry Performance (2016). Available in http://www.sindipecas.org.br. 